\title{
Carbon-ion radiotherapy for octogenarians with locally advanced non-small-cell lung cancer
}

\author{
Kazuhiko Hayashi ${ }^{1,2} \cdot$ Naoyoshi Yamamoto ${ }^{1}$ Mio Nakajima ${ }^{1} \cdot$ Akihiro Nomoto $^{1} \cdot$ Hitoshi Ishikawa ${ }^{1}$. \\ Kazuhiko Ogawa ${ }^{2} \cdot$ Hiroshi Tsuji $^{1}$
}

Received: 15 December 2020 / Accepted: 3 February 2021 / Published online: 19 February 2021

(c) The Author(s) 2021

\begin{abstract}
Purpose The clinical significance of carbon-ion radiotherapy (CIRT) for octogenarians with locally advanced non-small-cell lung cancer (LA-NSCLC) remains unclear. We aimed to evaluate the clinical outcomes of CIRT alone for octogenarians with LA-NSCLC.

Materials and methods We evaluated 32 patients who underwent CIRT alone between 1997 and 2015. The median age was 82.0 years (range, 80-88 years). In terms of clinical stage (UICC 7th edition), 7 (21.9\%), 10 (31.3\%), 11 (34.4\%), and $4(12.5 \%)$ patients had stage IIA, IIB, IIIA, and IIIB disease, respectively. The median CIRT dose was 72.0 Gy (relative biological effectiveness), and the median follow-up period was 33.1 months.

Results All patients successfully completed CIRT. Regarding grade $\geq 2$ toxicities, 1 (3.1\%), $3(9.4 \%)$, and $4(0.7 \%)$ patients developed grade 3 radiation pneumonitis, grade 2 radiation pneumonitis, and grade 2 dermatitis, respectively. No grade $\geq 4$ toxicities were observed. The 2 year LC, PFS, and OS rates were $83.5 \%, 46.7 \%$, and $68.0 \%$, respectively.

Conclusion CIRT alone is safe and effective for octogenarians with LA-NSCLC.
\end{abstract}

Keywords Carbon-ion radiotherapy $\cdot$ Locally advanced non-small-cell lung cancer $\cdot$ Octogenarians $\cdot$ Radiotherapy $\cdot$ Safety

\section{Introduction}

Primary lung cancer is one of the most common cancers worldwide [1]. Japan has one of the fastest aging societies, with a mean life expectancy at birth of 81 years for men and 87 years for women in 2017 [2]. Therefore, almost $40 \%$ of deaths owing to primary lung cancer are in octogenarians who are rarely eligible for highly invasive treatment modalities [3]. The standard treatment options for locally advanced non-small-cell lung cancer (LA-NSCLC) generally comprise surgery, chemotherapy, and/or radiotherapy. For inoperable patients, platinum-based chemotherapy or platinum-based chemoradiotherapy (CRT) is used. However,

Naoyoshi Yamamoto

yamamoto.naoyoshi@qst.go.jp

1 QST Hospital, National Institutes for Quantum and Radiological Sciences and Technology, 4-9-1 Anagawa, Inage-ku, Chiba 263-8555, Japan

2 Department of Radiation Oncology, Osaka University Graduate School of Medicine, 2-2 Yamadaoka, Suita, Osaka, Japan it sometimes induces severe hematologic toxicity, infection, esophagitis, and pneumonitis [4-6], thus making it highly toxic for octogenarians. The standard treatment modality for octogenarians with LA-NSCLC is controversial because there are limited data describing the specific therapies for patients aged 80 years or older. CRT, radiotherapy alone, and chemotherapy including an epidermal growth factor receptor tyrosine kinase inhibitor (EGFR-TKI) can be administered as treatment options [6]. In clinical practice, the treatment for octogenarians with LA-NSCLC is decided based on their performance status, comorbidity, and the patient value.

Carbon-ion radiotherapy (CIRT) is a high linear energy transfer radiotherapy that is used in some countries across Europe and Asia. CIRT has good dose-localizing properties [7], and thus can deliver a higher dose to the target volume than conventional photon radiotherapy while avoiding irradiation to the adjacent critical organs at risk, such as the lung, esophagus, trachea, and heart. CIRT alone can clinically achieve high local control (LC) rates with low toxicity [8-13]. Two published studies from our hospital have reported 2 year LC and overall survival (OS) rates of 83.5-93.1\% and 51.9-68.0\%, respectively, with CIRT alone 
in patients with LA-NSCLC $[8,13]$. The incidence of grade 3-4 toxicities ranged from $3.2 \%$ to $4.9 \%$, and none of the evaluated patients experienced grade 5 toxicities. Karube et al. conducted a multicenter study on CIRT alone in 64 patients with LA-NSCLC and reported similar 2 year LC and OS rates of $81.8 \%$ and $62.2 \%$, respectively [9]. No grade $\geq 3$ toxicities were observed. These clinical outcomes indicated that CIRT alone may be a safe treatment option for LA-NSCLC. However, these studies evaluated patients of all ages together, and no previous study has focused on octogenarians. Therefore, the safety and efficacy of CIRT alone for octogenarians with LA-NSCLC remain to be clarified. As such, this study aimed to investigate the clinical outcomes of CIRT alone for octogenarians with LA-NSCLC.

\section{Material and methods}

\section{Study design and patients}

This single-center retrospective study was approved by the Institutional Review Board of our institution (16-027) and was conducted according to the tenets of the Helsinki Declaration and its later amendments. The subjects were octogenarians who underwent CIRT alone between June 1997 and December 2015 at our institution. They were identified from a previously reported prospective phase I/II study of 72 patients of all ages and a previously reported retrospective study of 69 patients of all ages who were deemed ineligible for the phase I/II study at our institution $[8,13]$. The studies have been described in detail previously $[8,13]$. The inclusion criteria were as follows: (1) histologically or clinically diagnosed LA-NSCLC of stages IIA to IIIB (the UICC's TNM 7th Classification) [14], (2) Eastern Cooperative Oncology Group performance status of 0-2, (3) measurable tumors, (4) inoperable or refusal of surgery, (5) definitive treatments, (6) no other active cancers, and (7) no history of radiotherapy to the concerned region. The exclusion criteria included lung tumors with suspected invasion to the trachea, great vessels, heart, or carina. Consequently, data of 32 patients (18 patients from the prospective phase I/II study and 12 patients from the retrospective study) who met the inclusion criteria were analyzed.

Tumor stage was evaluated using computed tomography (CT) imaging of the chest and whole abdomen, enhanced magnetic resonance imaging (MRI) of the brain, chest radiography, and blood tests. Bone scans or [18F]-fluorodeoxyglucose positron emission tomography combined with CT (18F-FDG PET/CT) were also performed. Histological or cytological diagnosis was confirmed via bronchoscopic biopsy, CT-guided biopsy, or sputum cytology in $28(87.5 \%)$ patients and via CT, radiography, and/or 18F-FDG PET/CT in the remaining patients.
We collected information on grade $\geq 2$ toxicities. Treatment-related toxicities were graded according to the National Cancer Institute's Common Terminology Criteria for Adverse Events (version 4.0) [15].

\section{Carbon-ion radiotherapy}

Patients were fixed using an individually tailored immobilization device (Moldcare; Alcare, Tokyo, Japan; Shellfitter; Kuraray, Osaka, Japan), and CT images were obtained in the supine or prone position using respiratory sensors to monitor the respiratory phase $[7,8]$. Target delineation was performed as previously reported $[8,13]$. The primary lung lesion and metastatic lymph nodes were contoured as the gross tumor volume (GTV) on CT images. The GTV with a $10 \mathrm{~mm}$ margin and any prophylactic lymph nodes (ipsilateral hilar and/or mediastinal lymph nodes) were defined as the clinical target volume (CTV). For N0 cases, prophylactic lymph nodes irradiation was omitted irrespective of T-stage. Planning target volume (PTV) was defined as the $\mathrm{CTV}+5 \mathrm{~mm}$ safety margin. In cases where the PTV was close to the organs at risk, the margins for creating PTV was reduced.

The prescribed dose ranged from 68.0 to 76.0 Gy [relative biological effectiveness (RBE)] in 12-16 fractions, 4 days per week. Their prescribed doses were converted to 96.9 to $115.0 \mathrm{~Gy}$ (RBE) as the biologically effective dose using $\alpha / \beta=10$. A dose escalation study conducted during our investigation showed the potential of short-course CIRT alone at 72 Gy (RBE) [8], and thus the recommended dose was fixed at 72 Gy (RBE) in 16 fractions. Subsequently, this dose was adopted for all remaining patients $(n=21$, $65.6 \%$ ). The total dose was applied to the isocenter, and it enclosed the PTV conformably, with a $95 \%$ isodose line. The patients with lymph node metastasis underwent metastatic and prophylactic lymph node irradiation at a median dose of 49.5 Gy (RBE) [8, 13, 16]. The following irradiation dose constraints were applied: main bronchus, $60 \mathrm{~Gy}$ (RBE); esophagus, 50 Gy (RBE); and spinal cord, 30 Gy (RBE). Irradiation was performed in $2-5$ fields with 250 or $290 \mathrm{meV}$ carbon ions.

Regarding chemotherapy, 2 patients were administered induction chemotherapy, but none of the 32 patients received concurrent or adjuvant chemotherapy.

\section{Follow-up}

After treatment, follow-up observations were performed at $1,3,6,9$, and 12 months, and every 3-6 months thereafter if serious complications had not occurred. Follow-up comprised chest $\mathrm{CT}$, chest radiography, and blood tests during each evaluation. Brain MRI or F18-FDG PET/CT was performed as necessary. 


\section{Statistical analyses}

LC, progression-free survival (PFS), and OS were calculated using the Kaplan-Meier method. LC was defined as the time interval between irradiation commencement date and the local tumor regrowth at the PTV date or the last follow-up. PFS was defined as the time interval between irradiation commencement date and the date of disease progression at any site, death from any cause, or the last follow-up. OS was defined as the time interval between the start of irradiation and death or the last follow-up.

All statistical analyses were conducted using JMP statistical software (version 14.0; SAS Institute Inc., Cary, NC, USA).

\section{Results}

\section{Patient characteristics}

All patients successfully completed CIRT. The patient characteristics are summarized in Table 1. The median age was 82.0 years (range $80-88$ years), and the median follow-up period was 33.1 months (range 2.3-150.1 months) in the overall cohort and 36.9 months for survivors. This study included $28(87.5 \%)$ patients with performance status $1-2$. There were 3 (9.4\%), 12 (37.5\%), 12 (37.5\%), and 5 (15.6\%) patients who had T1, T2, T3, and T4 disease, respectively. Furthermore, 12 (37.5\%), 11 (34.4\%), and 9 (28.1\%) patients had N0, N1, and N2 disease, respectively. Seven patients (21.9\%) had stage IIA disease; 10 (31.3\%), stage IIB; 11 (34.4\%), stage IIIA; and 4 (12.5\%), stage IIIB. The median dose was 72.0 Gy (RBE).

\section{Toxicities}

Regarding grade $\geq 2$ lung toxicities, three (9.4\%) and one (3.1\%) patient developed grade 2 and grade 3 radiation pneumonitis, respectively (Table 2 ). In addition, four (0.7\%) patients developed grade 2 dermatitis. No grade $\geq 2$ toxicities other than dermatitis and pneumonitis were observed. Neither grade $\geq 4$ nor cardiac toxicities were observed.

\section{Local control and survival}

By the end of follow-up, 8 and 17 patients had died of cancer and unrelated causes, respectively, while 7 patients were still alive. At the time of first relapse, 3 local recurrences, 5 regional recurrences (regional lymph nodes), and 4 distant metastases were detected.

The 2 and 3 year LC rates were $83.5 \%$ [95\% confidence interval (CI) 62.9-93.8\%] and 77.1\% (95\% CI 54.3-90.5\%), respectively (Fig. 1a). The 2 and 3 year PFS
Table 1 Patient characteristics $(n=32)$

\begin{tabular}{|c|c|}
\hline Factors & Value \\
\hline \multicolumn{2}{|l|}{ Age (years) } \\
\hline Median & 82.0 \\
\hline Range & $(80.0-88.0)$ \\
\hline \multicolumn{2}{|l|}{ Sex } \\
\hline Male & $27(84.4)$ \\
\hline Female & $5(15.6)$ \\
\hline \multicolumn{2}{|l|}{ PS } \\
\hline 0 & $4(12.5)$ \\
\hline 1 & $26(81.3)$ \\
\hline 2 & $2(6.2)$ \\
\hline \multicolumn{2}{|l|}{ Smoking status } \\
\hline Current or previous & $27(84.4)$ \\
\hline Never & $5(15.6)$ \\
\hline \multicolumn{2}{|l|}{ Treatment status } \\
\hline Initial treatment & $30(93.8)$ \\
\hline Recurrence or residual cancer after chemotherapy & $2(6.2)$ \\
\hline \multicolumn{2}{|l|}{ Location of primary tumor } \\
\hline Upper lobe & $24(75.0)$ \\
\hline Middle lobe & $1(3.1)$ \\
\hline Lower lobe & $7(2.2)$ \\
\hline \multicolumn{2}{|l|}{ Operability } \\
\hline Yes & $4(12.5)$ \\
\hline No & $28(87.5)$ \\
\hline \multicolumn{2}{|l|}{ Clinical $\mathrm{T}$ classification } \\
\hline 1 & $3(9.4)$ \\
\hline 2 & $12(37.5)$ \\
\hline 3 & $12(37.5)$ \\
\hline 4 & $5(15.6)$ \\
\hline \multicolumn{2}{|l|}{ Clinical N classification } \\
\hline 0 & $12(37.5)$ \\
\hline 1 & $11(34.4)$ \\
\hline 2 & $9(28.1)$ \\
\hline \multicolumn{2}{|l|}{ Clinical stage } \\
\hline IIA & 7 (21.9) \\
\hline IIB & $10(31.3)$ \\
\hline IIIA & $11(34.4)$ \\
\hline IIIB & $4(12.5)$ \\
\hline \multicolumn{2}{|l|}{ Histology of primary lung cancer } \\
\hline Adenocarcinoma & $15(46.9)$ \\
\hline Squamous cell carcinoma & $11(34.4)$ \\
\hline Large cell carcinoma & $1(3.1)$ \\
\hline Non-small-cell carcinoma & $1(3.1)$ \\
\hline Unknown & $4(12.5)$ \\
\hline \multicolumn{2}{|l|}{ Irradiated field } \\
\hline Primary lesion & $13(40.6)$ \\
\hline Primary lesion + hilar lymph nodes & $4(12.5)$ \\
\hline Primary lesion + mediastinal lymph node & $15(46.9)$ \\
\hline \multicolumn{2}{|l|}{ Total dose (Gy RBE) } \\
\hline Median & 72 \\
\hline Range & $68-76$ \\
\hline
\end{tabular}


Table 1 (continued)

\begin{tabular}{ll}
\hline Factors & Value \\
\hline $\mathrm{CTV}\left(\mathrm{cm}^{3}\right)$ & \\
Median & 263.8 \\
Range & $80.9-1475.5$ \\
VC $\left(\mathrm{cm}^{3}\right)$ & \\
Median & 2300 \\
Range & $1100-3900$ \\
FEV1.0 $\left(\mathrm{cm}^{3}\right)$ & \\
Median & 1300 \\
Range & $600-2700$ \\
FEV1/FVC $(\%)$ & \\
Median & 63.8 \\
Range & $34.2-83.7$ \\
\%DLC & \\
Median & 75.5 \\
Range & $32.8-178.4$ \\
\hline
\end{tabular}

$P S$ performance status, $R B E$ relative biological effectiveness, $C T V$ clinical target volume, $V C$ vital capacity, $F E V 1$ forced expiratory volume in $1 \mathrm{~s}, F V C$ forced vital capacity, $\% D L C O$ percent of diffusing capacity for carbon monoxide

Table 2 Treatment-related toxicities

\begin{tabular}{lllll}
\hline Grade & $2(\%)$ & $3(\%)$ & $4(\%)$ & Total $(\%)$ \\
\hline Dermatitis & $4(12.5)$ & 0 & 0 & $4(12.5)$ \\
Pneumonitis & $3(9.4)$ & $1(3.1)$ & 0 & $4(12.5)$ \\
\hline
\end{tabular}

rates were $46.7 \%$ (95\% CI 32.9-68.8\%) and $41.5 \%$ (95\% CI 29.2-61.2\%), respectively (Fig. 1b). The 2 and 3 year OS rates were $68.0 \%$ (95\% CI 50.0-81.8\%) and $54.3 \%$ (95\% CI $36.7-70.8 \%$ ), respectively (Fig. 1c). The median PFS and OS durations were 20.8 and 33.1 months, respectively.

For survival analysis according to clinical stage, the participants were categorized into those with stages II and III LA-NSCLC, and the corresponding median PFS rates were 21.6 months and 15.1 months. Meanwhile, the corresponding median OS rates were 45.9 months and 22.2 months, respectively. In addition, the 2 year LC, PFS, and OS rates of patients with stage II disease were $93.3 \%, 55.8 \%$, and $82.4 \%$, whereas those with stage III disease were $70.7 \%, 44.4 \%$, and $50.6 \%$, respectively.

\section{Discussion}

CRT as a treatment option for octogenarians with LANSCLC induces several toxicities. However, photon radiotherapy also yields unsatisfactory outcomes, and the adoption of EGFR-TKI is rather limited. Therefore, other treatment approaches that are safer and more effective are needed for these patients. The results of this study show that CIRT alone is safe and effective for octogenarians with LA-NSCLC. To our best knowledge, this is the first study to report the clinical usefulness of CIRT alone for octogenarians with LA-NSCLC to date.

Only one study has focused on treatment options for octogenarians with LA-NSCLC [17]. In the study by Corre et al. octogenarians with EGFR-mutated stage I-IV lung cancer were treated using EGFR-TKI (Table 3). The main grade $\geq 3$ toxicities were diarrhea (17.0\% of patients), cutaneous $(10.3 \%)$, and others $(41.0 \%)$. With respect to photon CRT or radiotherapy alone, only studies in septuagenarians were conducted. Atagi et al. conducted a phase III trial of photon radiotherapy with or without low-dose carboplatin for patients older than 70 years [median age 77 years (range 71-93 years)] with stage III LA-NSCLC [18]. The most common grade $\geq 3$ toxicity in the CRT group was leukopenia (63.5\%), followed by thrombocytopenia (29.2\%), infection (12.5\%), radiation pneumonitis (6.5\%), and dyspnea (4.2\%). In contrast, the most common grade $\geq 3$ toxicity in the photon radiotherapy alone group was radiation pneumonitis (5.3\%), closely followed by dyspnea (5.1\%) and infection (4.1\%). Meanwhile, in our study, grade 3 radiation pneumonitis occurred in only $3.1 \%$ of all patients, and none of the patients developed other grade $\geq 3$ toxicities. These findings indicate that CIRT alone may be safer than photon CRT or chemotherapy, including EGFR-TKI, and approximately comparable to photon radiotherapy alone with respect to grade $\geq 3$ toxicity.

Several studies on photon radiotherapy alone for patients older than 70 years with stage III LA-NSCLC reported that the median PFS and OS of radiotherapy alone were 6.8 months and 11-18.1 months, respectively [18-21]. Driessen et al. reported a median OS of 5 months when patients older than 70 years with stage III LA-NSCLC selected non-curative treatment [19]. Meanwhile, although our study included only patients with N0-2 metastatic lymph node status, and consequently did not include all stage III patients, the median PFS and OS for stage III LA-NSCLC were 15.1 and 22.2 months, respectively, despite the median age being 82.0 years. Further, all the patients completed CIRT on schedule. These findings indicate that CIRT alone would be an effective treatment option for patients classified as $\mathrm{N} 0-2$.

The side effects of definitive treatment are generally more severe in elderly patients than in younger patients. Therefore, for elderly patients, the clinical goals must be decided by considering not only survival, but also quality of life and treatment tolerance. Administration of EGFRTKI is limited to patients with NSCLC with activating EGFR mutation, although it is effective and relatively safe [22]. In addition, sustained disease control is uncommon [22]. Further, while CRT is effective, it is highly toxic 

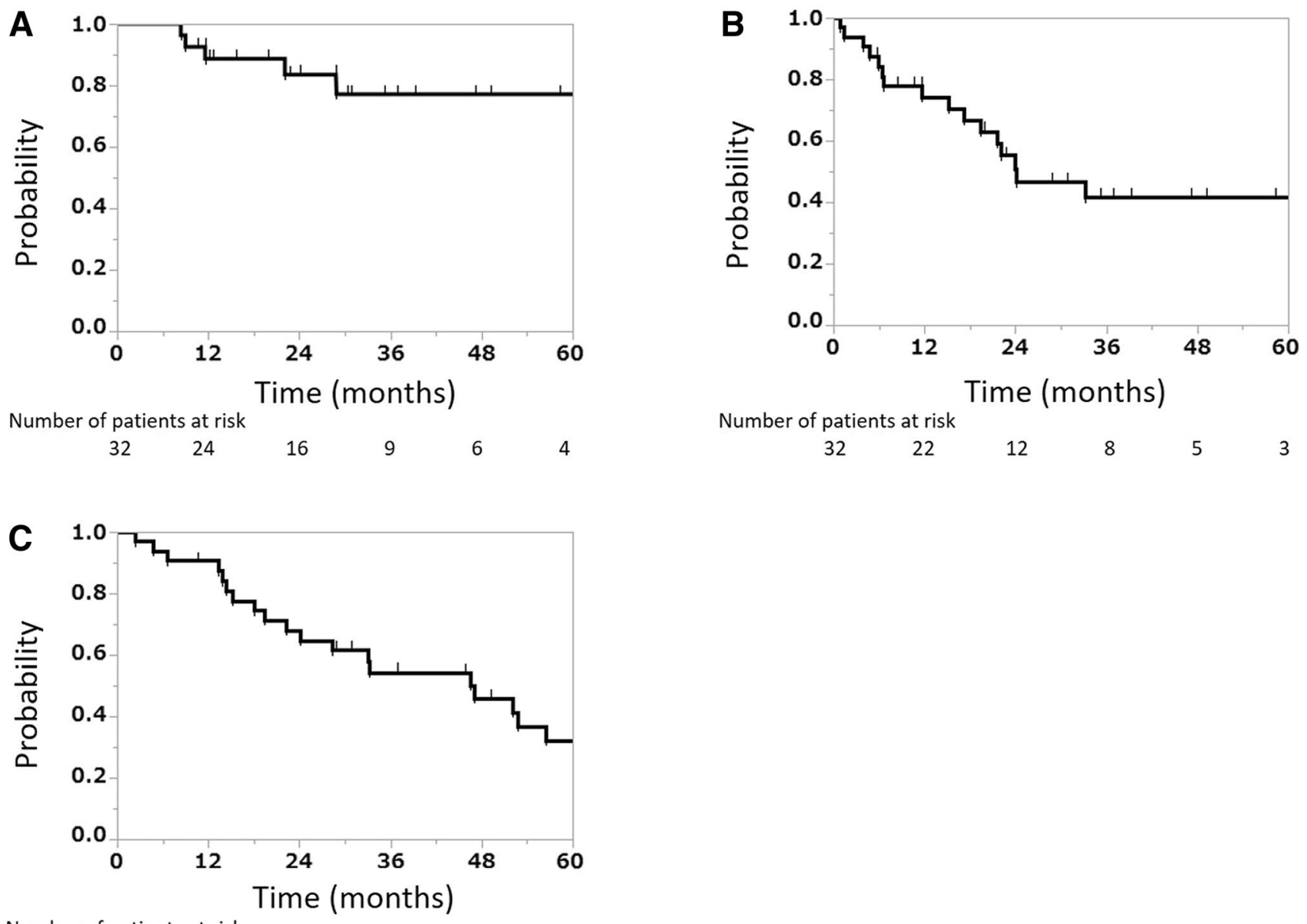

Number of patients at risk

$\begin{array}{llllll}32 & 29 & 22 & 16 & 12 & 8\end{array}$

Fig. 1 Local control rate (a), progression-free survival rate (b), and overall survival rate (c)

for octogenarians and may worsen quality of life [21]. Unfortunately, photon radiotherapy alone also yields poor prognosis despite the relatively low incidence of severe toxicity. Meanwhile, CIRT alone achieved both a low incidence of severe toxicities and favorable local control, thus making it a promising treatment option for octogenarians with LA-NSCLC.

Our study had several limitations. First, the present study was a single-center retrospective analysis. Second, our results were derived from a small sample size of only 32 patients. Third, only octogenarians with Eastern Cooperative Oncology Group performance status of $0-2$ were treated with CIRT alone, and thus there might have been patient bias. Finally, the total doses and fractionation varied (68-76 Gy (RBE) in 12-16 fractions) among the patients. Further large-scale multicenter prospective trials are warranted.

In conclusion, CIRT alone is a relatively safe and effective treatment modality for octogenarians with LA-NSCLC. 


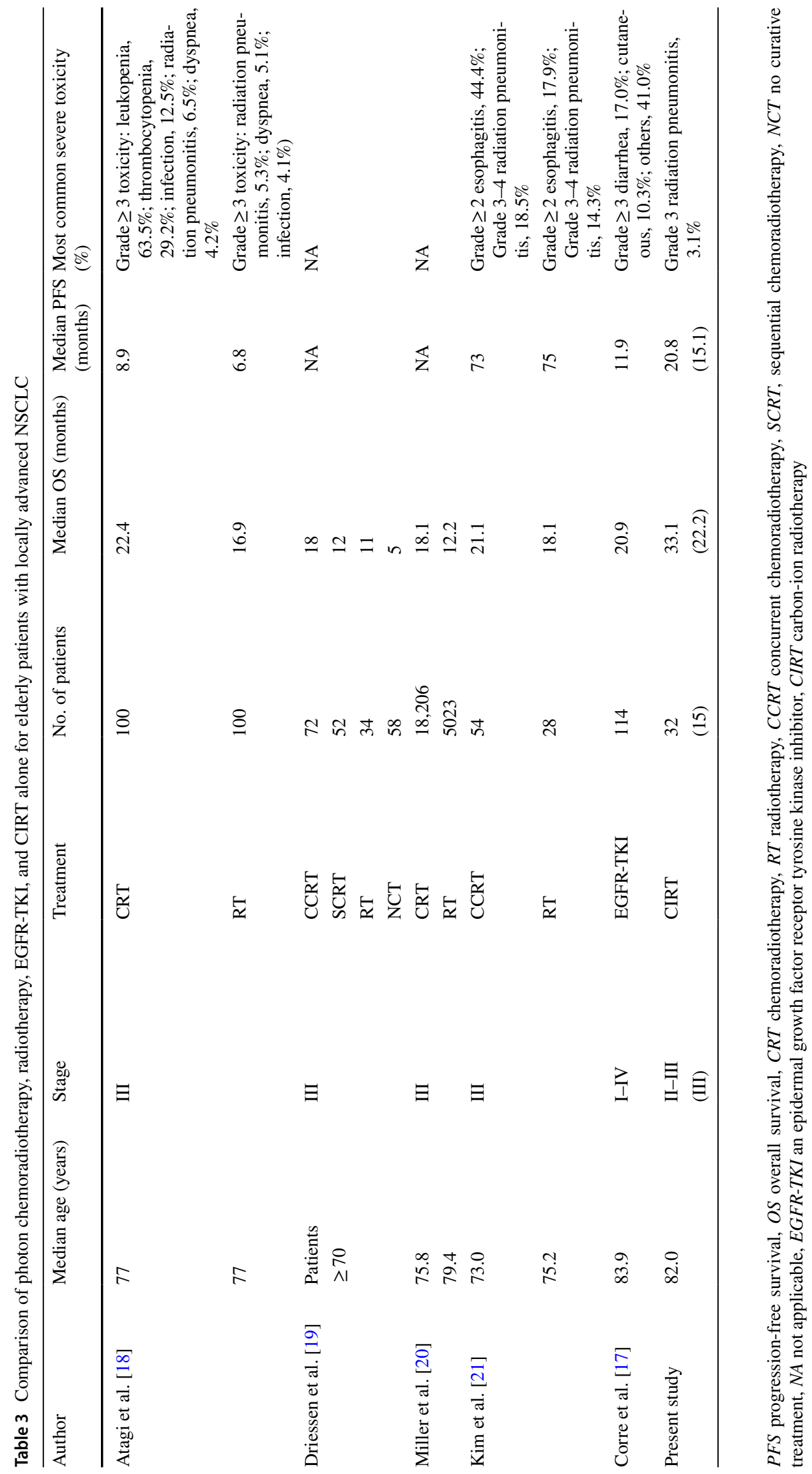


Acknowledgements We thank the members of the Our Institution Working Group for Lung Cancer. This research did not receive any specific grant from funding agencies in the public, commercial, or notfor-profit sectors.

Funding No funding was received.

\section{Compliance with ethical standards}

Conflict of interest The authors have no conflict of interest.

Open Access This article is licensed under a Creative Commons Attribution 4.0 International License, which permits use, sharing, adaptation, distribution and reproduction in any medium or format, as long as you give appropriate credit to the original author(s) and the source, provide a link to the Creative Commons licence, and indicate if changes were made. The images or other third party material in this article are included in the article's Creative Commons licence, unless indicated otherwise in a credit line to the material. If material is not included in the article's Creative Commons licence and your intended use is not permitted by statutory regulation or exceeds the permitted use, you will need to obtain permission directly from the copyright holder. To view a copy of this licence, visit http://creativecommons.org/licenses/by/4.0/.

\section{References}

1. de Groot PM, Wu CC, Carter BW, Munden RF. The epidemiology of lung cancer. Transl Lung Cancer Res. 2018;7:220-33.

2. Statistics of Japan. http://www.e-stat.go.jp/en. Published 2017.

3. Cancer Information Service. http://gdb.ganjoho.jp/graph_db/ gdb1 ?dataType $=20 \&$ graphId $=101 \&$ total Target $=11 \&$ useLo $\mathrm{g}=$ on\&_stackedRaito=on\&_showErrorBar=on\&_useUnknown Stage $=$ on\&_smTypes $=$ on \&_smTypes $=$ on \&_smTypes $=$ on \& smTypes $=$ on \&_smTypes $=$ on\&_smTypes $=$ on \&_smTypes $=$ on $\&$ _ smTypes=on\&_smTypes=on\&_smType. Published 2018.

4. Oh IJ, Ahn SJ. Multidisciplinary team approach for the management of patients with locally advanced non-small cell lung cancer: searching the evidence to guide the decision. Radiat Oncol J. 2017;35:16-24.

5. Schild SE, Stella PJ, Geyer SM, Bonner JA, McGinnis WL, Mailliard JA, et al. The outcome of combined-modality therapy for stage III non-small-cell lung cancer in the elderly. J Clin Oncol. 2003;21:3201-6.

6. Gajra A, Jatoi A. Non-small-cell lung cancer in elderly patients: a discussion of treatment options. J Clin Oncol. 2014;32:2562-9.

7. Kanai T, Endo M, Minohara S, Miyahara N, Koyama-ito H, Tomura H, et al. Biophysical characteristics of HIMAC clinical irradiation system for heavy-ion radiation therapy. Int J Radiat Oncol Biol Phys. 1999;44(4):201-10.https://doi.org/10.1016/ s0360-3016(98)00544-6.

8. Takahashi W, Nakajima M, Yamamoto N, Yamashita H, Nakagawa K, Miyamoto T, et al. A prospective nonrandomized phase I/II study of carbon ion radiotherapy in a favorable subset of locally advanced non-small cell lung cancer (NSCLC). Cancer. 2015;121:1321-7.

9. Karube M, Yamamoto N, Shioyama Y, Saito J, Matsunobu J, Okimoto J, et al. Carbon-ion radiotherapy for patients with advanced stage non-small-cell lung cancer at multicenters. J Radiat Res. 2017;58:761-4.

10. Yamamoto N, Miyamoto T, Nishimura H, Koto M, Tsujii H, Ohwada $\mathrm{H}$, et al. Preoperative carbon ion radiotherapy for nonsmall cell lung cancer with chest wall invasion-pathological findings concerning tumor response and radiation induced lung injury in the resected organs. Lung Cancer. 2003;42:87-95.

11. Sugane T, Baba M, Imai R, Nakajima M, Yamamoto N, Miyamoto $\mathrm{T}$, et al. Carbon ion radiotherapy for elderly patients 80 years and older with stage I non-small cell lung cancer. Lung Cancer. 2009;64:45-50.

12. Anzai M, Yamamoto N, Hayashi K, Nakajima M, Nomoto A, Ogawa K, et al. Safety and efficacy of carbon-ion radiotherapy alone for stage III non-small cell lung cancer. Anticancer Res. 2020;40:379-86.

13. Hayashi K, Yamamoto N, Nakajima M, Nomoto A, Tsuji H, Ogawa $\mathrm{K}$, et al. Clinical outcomes of carbon-ion radiotherapy for locally advanced non-small-cell lung cancer. Cancer Sci. 2019;110:734-41.

14. Sobin LH, Gospodarowicz MK, Wittekind C, editors. International union against cancer. TNM classification of malignant tumours. 7th ed. New York: Wiley-Blackwell Inc; 2009.

15. Services UDOHAH, ed. Common terminology criteria for adverse events v4.0. NIH publication; 2009. https://evs.nci.nih.gov/ftp1/ CTCAE/CTCAE_4.03_2010-06-14_\%0AQuickReference_5x7. pdf.

16. Hayashi K, Yamamoto N, Karube M, Nakajima M, Matsufuji $\mathrm{N}$, Tsuji $\mathrm{H}$, et al. Prognostic analysis of radiation pneumonitis: carbon-ion radiotherapy in patients with locally advanced lung cancer. Radiat Oncol. 2017;12:91.

17. Corre R, Gervais R, Guisier F, Tassy L, Vinas F, Lamy R, et al. Octogenarians with EGFR-mutated non-small cell lung cancer treated by tyrosine-kinase inhibitor: a multicentric real-world study assessing tolerance and efficacy (OCTOMUT study). Oncotarget. 2018;9:8253-62.

18. Atagi S, Kawahara M, Yokoyama A, Okamoto H, Yamamoto N, Ohe Y, et al. Thoracic radiotherapy with or without daily low-dose carboplatin in elderly patients with non-small-cell lung cancer: a randomized, controlled, phase 3 trial by the Japan Clinical Oncology Group (JCOG0301). Lancet Oncol. 2012;13:671-8.

19. Driessen EJM, Bootsma GP, Hendriks LEL, van den Berkmortel FW, Bogaarts BA, van Loon JG, et al. Stage III non-small cell lung cancer in the elderly: patient characteristics predictive for tolerance and survival of chemoradiation in daily clinical practice. Radiother Oncol. 2016;121:26-31.

20. Miller ED, Fisher JL, Haglund KE, Grecula JC, Xu-Welliver M, Bertino EM, et al. The addition of chemotherapy to radiation therapy improves survival in elderly patients with stage III non-small cell lung cancer. J Thorac Oncol. 2018;13:426-35.

21. Kim DY, Song C, Kim SH, Kim YJ, Lee JS, Kim JS. Chemoradiotherapy versus radiotherapy alone following induction chemotherapy for elderly patients with stage iii lung cancer. Radiat Oncol J. 2019;37:176-84.

22. Bunn PA, Dimou A. Systemic therapy for elderly patients with advanced non-small-cell lung cancers. J Clin Oncol. 2018;36:2571-4.

Publisher's Note Springer Nature remains neutral with regard to jurisdictional claims in published maps and institutional affiliations. 